\title{
Judgment aggregation with consistency alone
}

Citation for published version (APA):

Dietrich, F. K., \& List, C. (2007). Judgment aggregation with consistency alone. METEOR, Maastricht University School of Business and Economics. METEOR Research Memorandum No. 021 https://doi.org/10.26481/umamet.2007021

Document status and date:

Published: 01/01/2007

DOI:

10.26481/umamet.2007021

Document Version:

Publisher's PDF, also known as Version of record

\section{Please check the document version of this publication:}

- A submitted manuscript is the version of the article upon submission and before peer-review. There can be important differences between the submitted version and the official published version of record.

People interested in the research are advised to contact the author for the final version of the publication, or visit the DOI to the publisher's website.

- The final author version and the galley proof are versions of the publication after peer review.

- The final published version features the final layout of the paper including the volume, issue and page numbers.

Link to publication

\footnotetext{
General rights rights.

- You may freely distribute the URL identifying the publication in the public portal. please follow below link for the End User Agreement:

www.umlib.nl/taverne-license

Take down policy

If you believe that this document breaches copyright please contact us at:

repository@maastrichtuniversity.nl

providing details and we will investigate your claim.
}

Copyright and moral rights for the publications made accessible in the public portal are retained by the authors and/or other copyright owners and it is a condition of accessing publications that users recognise and abide by the legal requirements associated with these

- Users may download and print one copy of any publication from the public portal for the purpose of private study or research.

- You may not further distribute the material or use it for any profit-making activity or commercial gain

If the publication is distributed under the terms of Article $25 \mathrm{fa}$ of the Dutch Copyright Act, indicated by the "Taverne" license above, 
Franz Dietrich, Christian List

Judgment aggregation with consistency alone

$\mathrm{RM} / 07 / 021$

JEL code: D70, D71

\section{METE@R

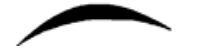

Maastricht research school of Economics

of TEchnology and ORganizations

Universiteit Maastricht

Faculty of Economics and Business Administration

P.O. Box 616

NL - 6200 MD Maastricht

phone : ++31433883830

fax $\quad:++31433884873$ 



\title{
Judgment aggregation with consistency alone
}

\author{
Franz Dietrich and Christian List ${ }^{1}$
}

30 May 2007

\begin{abstract}
All existing impossibility theorems on judgment aggregation require individual and collective judgment sets to be consistent and complete (in some recent results with completeness relaxed to deductive closure), arguably a demanding rationality requirement. They do not carry over to aggregation functions mapping profiles of (merely) consistent individual judgment sets to (merely) consistent collective ones. We prove that, whenever the agenda of propositions under consideration exhibits mild interconnections, any such aggregation function that is "neutral" between the acceptance and rejection of each proposition is dictatorial. We relate this theorem to the literature.
\end{abstract}

\section{Introduction}

There are now many impossibility results on the aggregation of individual judgments on logically connected propositions (such as $a, a \rightarrow b$ and $b$ ) into collective judgments. Sparked by the observation that majority voting fails to guarantee rational collective judgments for some profiles of individual judgments (as illustrated in table 1, where majority voting in a three-member group leads to inconsistent collective judgments), several authors have shown that any aggregation function that has certain properties in common with majority voting does so too (List and Pettit 2002, 2004; Pauly and van Hees 2006; Dietrich 2006, 2007; Gärdenfors 2006; Nehring and Puppe 2002, 2005, 2006; van Hees 2007; Mongin 2005; Dietrich and List 2005, 2006, 2007a; Dokow and Holzman 2005, 2006; the literature goes back to the "doctrinal" and "discursive paradoxes" in Kornhauser and Sager 1986 and Pettit 2001).

\begin{tabular}{|c|c|c|c|}
\hline & $a$ & $a \rightarrow b$ & $b$ \\
\hline Individual 1 & True & True & True \\
\hline Individual 2 & True & False & False \\
\hline Individual 3 & False & True & False \\
\hline Majority & True & True & False \\
\hline
\end{tabular}

Table 1: A majority inconsistency

\footnotetext{
${ }^{1}$ Addresses: C. List, Department of Government, London School of Economics, London WC2A 2AE, U.K.; F. Dietrich, Department of Quantitative Economics, University of Maastricht, P.O. Box 616, 6200 MD Maastricht, The Netherlands.
} 
Almost all of these impossibility results formalize rationality as the conjunction of two requirements. Individual and collective judgments must be both logically consistent and complete, i.e., the set of accepted propositions must not entail a contradiction (consistency) and it must contain a member of every proposition-negation pair under consideration (completeness). This is a strong reqirement, whose completeness part, in particular, has been criticized as being too demanding in many real-world decision-making settings (see especially Gärdenfors 2006, but also List and Pettit 2002, Dietrich and List 2007b, Goodin and List 2006). Often individuals and groups wish to abstain from making any judgment on certain proposition-negation pairs. Courts and expert panels, for example, may wish to abstain from making judgments on issues on which there is too much uncertainty, and legislatures and international decision-making bodies, such as the EU Council of Ministers or the UN Security Council, on issues on which there is too much disagreement.

But while some recent impossibility results (Gärdenfors 2006, Dietrich and List 2006, Dokow and Holzman 2006) give up the completeness requirement in favour of deductive closure - i.e., any proposition under consideration that is entailed by other accepted propositions must also be accepted - it is so far an open question whether judgment aggregation faces any serious impossibilities if only consistency is required. This question is interesting, since consistency is the most natural and least contentious requirement of rationality. One may not expect compelling impossibility results if consistency is the only rationality requirement: all of the standard conditions on judgment aggregation functions (including independence/systematicity and only excluding completeness and deductive closure) are satisfied, for example, by suitable (symmetrical) supermajority rules (for details, see section 4). Thus these standard conditions do not lead to any impossibilities in the case of consistency alone.

However, we here introduce a new condition, acceptance/rejection neutrality, and show that, if (and only if) the agenda of propositions under consideration satisfies a mild condition, any acceptance/rejection neutral aggregation function that maps consistent individual judgments to consistent collective ones is dictatorial. Acceptance/rejection neutrality requires the aggregation function not to be biased either for or against the acceptance of any proposition. This condition is a variant of May's (1952) neutrality condition on a single binary choice and the conditions of neutrality-within-issues in Nehring and Puppe (2005) and unbiasedness in Dietrich and List (2005). Although our present result is the first one with the weak rationality requirement of consistency alone, it is mathematically related to the results of the latter two sets of authors using neutrality-withinissues or unbiasedness under the "full" rationality requirement of consistency and completeness. But the possible incompleteness of judgments in our result creates subtleties in defining neutrality that do not arise when judgments are complete, as explained below. In particular, the earlier definitions of neutrality are unsuitable for obtaining our present result. 
Our result should be interpreted as a baseline result. One may not always wish to demand acceptance/rejection neutrality. However, it is surprising that, under the present weak rationality requirement, this single condition on the aggregation function suffices to characterize dictatorships. Below we also briefly discuss the relationship of our result with the literature on belief merging in computer science (e.g., Konieczny and Pino-Perez 2002).

\section{The model}

We consider a group of individuals $N=\{1,2, \ldots, n\}(n \geq 2)$ making judgments on some propositions represented in logic (Dietrich 2007, generalizing List and Pettit 2002, 2004).

Logic. To define a logic, let $\mathbf{L}$ be a set of sentences, called propositions, closed under negation (i.e., if $p \in \mathbf{L}$ then $\neg p \in \mathbf{L}$, where $\neg$ is the negation symbol), and stipulate that each subset $S \subseteq \mathbf{L}$ is either consistent or inconsistent, subject to standard axioms. ${ }^{2}$ In standard propositional logic, for example, $\mathbf{L}$ contains propositions such as $a, b, a \wedge b, a \vee b, \neg(a \rightarrow b)$ (where $\wedge, \vee, \rightarrow$ denote "and", "or", "if-then", respectively); sets such as $\{a, a \rightarrow b, b\}$ and $\{a \wedge b\}$ are consistent, while sets such as $\{a, \neg a\}$ and $\{a, a \rightarrow b, \neg b\}$ are inconsistent.

Agenda. The agenda is the set of propositions on which judgments are to be made, defined as a non-empty subset $X \subseteq \mathbf{L}$ expressible as $X=\{p, \neg p: p \in$ $\left.X_{+}\right\}$for a set $X_{+} \subseteq \mathbf{L}$ of unnegated propositions. Double negations cancel each other out, i.e., $\neg \neg p$ stands for $p .^{3}$ In the example in table $1, X$ is $\{a, \neg a, a \rightarrow$ $b, \neg(a \rightarrow b), b, \neg b\}$ in standard propositional logic (or in a conditional logic). A subset $Y \subseteq X$ that forms an agenda itself (i.e., is non-empty and expressible as $\left\{p, \neg p: p \in Y_{+}\right\}$with $Y_{+}$containing unnegated propositions) is called a sub-agenda of $X$.

Individual judgment sets. Each individual $i$ 's judgment set is the set $A_{i} \subseteq$ $X$ of propositions that he or she accepts (e.g., believes to be true). A profile is an $n$-tuple $\left(A_{1}, \ldots, A_{n}\right)$ of individual judgment sets. Each judgment set may or may not be consistent.

\footnotetext{
${ }^{2} \mathrm{C} 1$ : For any $p \in \mathbf{L},\{p, \neg p\}$ is inconsistent. C2: If $S \subseteq \mathbf{L}$ is inconsistent, then so is any superset $T \supseteq S$ (in $\mathbf{L}$ ). C3: $\emptyset$ is consistent, and each consistent $S \subseteq \mathbf{L}$ has a consistent superset $T \supseteq S$ (in $\mathbf{L}$ ) containing a member of each pair $p, \neg p \in \mathbf{L}$. C4: Any inconsistent set $S \subseteq \mathbf{L}$ has a finite inconsistent subset $T \subseteq S$. For details, see Dietrich (2007).

${ }^{3}$ Strictly speaking, when we use the negation symbol $\neg$ hereafter, we mean a modified negation symbol $\sim$, where $\sim p:=\neg p$ if $p$ is not a negated proposition and $\sim p:=q$ if $p=\neg q$ for some $q$.
} 
Aggregation functions. An aggregation function is a function $F$ that maps each profile $\left(A_{1}, \ldots, A_{n}\right)$ in some domain of admissible ones to a collective judgment set $F\left(A_{1}, \ldots, A_{n}\right)=A \subseteq X$, interpreted as the set of propositions that the group as a whole accepts. An aggregation function is consistent if it produces a consistent collective judgment set as output. It has full domain if the admissible profiles are all profiles of consistent individual judgment sets. This domain is larger than the so-called universal domain, which additionally requires completeness of individual judgment sets.

Examples of aggregation functions are majority voting, where for each profile of consistent individual judgment sets $\left(A_{1}, \ldots, A_{n}\right)$,

$$
F\left(A_{1}, \ldots, A_{n}\right)=\left\{p \in X:\left|\left\{i \in N: p \in A_{i}\right\}\right|>\left|\left\{i \in N: p \notin A_{i}\right\}\right|\right\},
$$

and a dictatorship of individual $i \in N$, where, for each such $\left(A_{1}, \ldots, A_{n}\right)$,

$$
F\left(A_{1}, \ldots, A_{n}\right)=A_{i}
$$

Majority voting has full domain but is not consistent, as noted above. Dictatorships, by contrast, have full domain and are consistent. We also call an aggregation function a dictatorship (of individual $i \in N$ ) on a sub-agenda $Y \subseteq X$, if, for each profile $\left(A_{1}, \ldots, A_{n}\right), F\left(A_{1}, \ldots, A_{n}\right) \cap Y=A_{i} \cap Y$.

\section{Result}

To state our result, we need to introduce its condition on the agenda and its condition on the aggregation function.

The condition on the agenda. The agenda $X$ is non-simple if it has a minimal inconsistent subset $S \subseteq X$ of three or more propositions (where an inconsistent set is minimal if every proper subset is consistent); $X$ is nonseparable if it cannot be partitioned into two sub-agendas $X_{1}$ and $X_{2}$ such that $S_{1} \cup S_{2}$ is consistent for any consistent subsets $S_{1} \subseteq X_{1}$ and $S_{2} \subseteq X_{2}$. For example, the agenda $X=\{a, \neg a, a \rightarrow b, \neg(a \rightarrow b), b, \neg b\}$ is non-simple (take $S=\{a, a \rightarrow b, \neg b\}$ ) and non-separable (as easily seen). The (trivial) agenda $X=\{a, \neg a, b, \neg b\}$ is neither non-simple (its largest minimal inconsistent subsets are $\{a, \neg a\}$ and $\{b, \neg b\}$ ) nor non-separable (take $X_{1}=\{a, \neg a\}$ and $\left.X_{2}=\{b, \neg b\}\right)$.

The condition on the aggregation function. An aggregation function $F$ is acceptance/rejection neutral if, for any proposition $p \in X$ and admissible profiles $\left(A_{1}, \ldots, A_{n}\right),\left(A_{1}^{*}, \ldots, A_{n}^{*}\right)$,

[for all $\left.i \in N, p \in A_{i} \Leftrightarrow p \notin A_{i}^{*}\right] \Rightarrow\left[p \in F\left(A_{1}, \ldots, A_{n}\right) \Leftrightarrow p \notin F\left(A_{1}^{*}, \ldots, A_{n}^{*}\right)\right]$. 
Acceptance/rejection neutrality is inspired by May's (1952) condition of neutrality in a binary choice. It requires that if the individuals accepting some proposition $p$ in one profile are the same as those rejecting $p$ in another, then $p$ is collectively accepted in the first profile if and only if it is collectively rejected in the second. Informally, as noted above, this requires the aggregation function not to be biased either for or against the acceptance of any proposition. Majority voting (for odd group size) and dictatorships are clearly acceptance/rejection neutral. (These examples are quite special: they use the same decision criterion for each proposition, although not required by acceptance/rejection neutrality.) Under the full rationality requirement of consistency and completeness, acceptance/rejection neutrality becomes equivalent to the earlier conditions of unbiasedness (Dietrich and List 2005) and (essentially) neutrality-within-issues (Nehring and Puppe 2005). Without full rationality, however, there are crucial differences between the present condition and those earlier conditions, as explained in the next section.

Theorem 1 If (and only if) the agenda is non-simple and non-separable, every consistent full-domain aggregation function satisfying acceptance/rejection neutrality is a dictatorship. ${ }^{4}$

Under the mild rationality requirement of consistency and the mild agenda condition of non-simplicity and non-separability, as satisfied by all the lead examples in the literature on judgment aggregation, acceptance/rejection neutrality thus fully characterizes the class of dictatorships. The following corollary, which applies to every agenda, is also of interest:

Corollary 1 Every consistent full-domain aggregation function satisfying acceptance/rejection neutrality is a dictatorship on every non-simple and nonseparable sub-agenda.

(In particular, since every non-simple agenda has at least one non-simple and non-separable sub-agenda, corollary 1 implies that, for any non-simple agenda, acceptance/rejection neutrality leads to a "local dictatorship".)

As noted in the introduction, our result (theorem 1 and corollary 1) can be seen as a "consistency-alone" analogue of earlier characterizations of ("global" and "local") dictatorships by Nehring and Puppe (2005) and Dietrich and List (2005) using neutrality-within-issues or unbiasedness under full rationality. In the appendix, we derive our result from Dietrich and List's result. Surprisingly, our new theorem, despite its weaker rationality requirement, requires fewer conditions on the aggregation function than the Nehring and Puppe result and equivalent conditions on the agenda as it; ${ }^{5}$ and it requires fewer conditions on

\footnotetext{
${ }^{4}$ The "only if" part requires $n \geq 3$.

${ }^{5}$ The result's additional aggregation condition is monotonicity (without it, there are counterexamples). The agenda conditions ("not a median space" and "not decomposable") can be shown to be equivalent to ours (non-simplicity and non-separability).
} 
the agenda than the Dietrich and List result and equivalent conditions on the aggregation function as it. ${ }^{6}$

\section{Discussion}

We have proved the first impossibility theorem on judgment aggregation under the rationality requirement of consistency alone. Why is this interesting? It is interesting not only because consistency is the least contentious requirement of rationality, but also because the result connects judgment aggregation with the important related body of literature on belief merging in computer science (Konieczny and Pino-Perez 2002, Pigozzi 2006). In belief merging, several consistent sets of propositions in some language have to be merged into a single consistent set. The individual sets need not be complete, and their elements need not be drawn from the same (small) agenda. This problem is equivalent to judgment aggregation with the agenda $X$ chosen to comprise the entire language $\mathbf{L}$ or a sufficiently large portion of it and consistency as the only rationality requirement. Conditions of propositionwise aggregation are not usually considered in that literature, but our present result provides an insight into what is needed to characterize dictatorships in a belief merging context.

Crucially, under the rationality requirement of consistency alone, none of the standard conditions on aggregation functions used in the literature - including the standard neutrality conditions - lead to an impossibility result. Consider the conditions of systematicity, unbiasedness, independence, monotonicity, the unanimity principle and anonymity. Systematicity requires that, for any pair of propositions $p, q \in X$ and admissible profiles $\left(A_{1}, \ldots, A_{n}\right),\left(A_{1}^{*}, \ldots, A_{n}^{*}\right)$,

[for all $\left.i \in N, p \in A_{i} \Leftrightarrow q \in A_{i}^{*}\right] \Rightarrow\left[p \in F\left(A_{1}, \ldots, A_{n}\right) \Leftrightarrow q \in F\left(A_{1}^{*}, \ldots, A_{n}^{*}\right)\right]$.

Unbiasedness and independence can be obtained from this definition by restricting the quantification over pairs of propositions $p, q \in X$ to propositionnegation pairs (i.e., $p=\neg q$ ) and to single propositions (i.e., $p=q$ ), respectively. Monotonicity requires that any additional individual support for a collectively accepted proposition does not reverse its collective acceptance, and the unanimity principle that any proposition accepted by all individuals is also collectively accepted. Anonymity, finally, requires invariance of the collective judgment set under permutations of the individual judgment sets and thus excludes aggregation functions that do not treat individuals equally. These conditions can be jointly satisfied, for any agenda, by a consistent (but of course not complete, nor generally deductively closed) aggregation function with full domain. An example is (symmetrical) supermajority voting, where for each profile of consistent

\footnotetext{
${ }^{6}$ The result's additional agenda conditions are "even-number negatability" and "local asymmetry" (without them, there are counterexamples). The aggregation condition (unbiasedness) is equivalent to ours (acceptance/rejection neutrality) under the stronger rationality requirement.
} 
individual judgment sets $\left(A_{1}, \ldots, A_{n}\right)$,

$$
F\left(A_{1}, \ldots, A_{n}\right)=\left\{p \in X:\left|\left\{i \in N: p \in A_{i}\right\}\right| / n \geq q\right\},
$$

with the acceptance quota $q$ chosen to be sufficiently large (List and Pettit 2002, Dietrich and List 2007b). The consistency of this aggregation function is easy to see for $q=1$ (in which case deductive closure is still met while completeness is violated), but it is enough to require $q>\frac{k-1}{k}$, where $k$ is the size of the largest minimal inconsistent subset of $X$ (in which case both deductive closure and completeness are violated, unless $q=1$ ). It is particularly important to note that symmetrical supermajority voting satisfies unbiasedness, i.e., the requirement of equal treatment of proposition-negation pairs, while violating acceptance/rejection neutrality. This illustrates that, although acceptance/rejection neutrality is equivalent to unbiasedness in the full rationality case, the two conditions come significantly apart under the requirement of consistency alone. While unbiasedness is innocuous here, acceptance/rejection neutrality leads to a dicatorship.

\section{References}

Dietrich, F. (2006) Judgment Aggregation: (Im)Possibility Theorems. Journal of Economic Theory 126(1): 286-298

Dietrich, F. (2007) A generalised model of judgment aggregation. Social Choice and Welfare 28(4): 529-565

Dietrich, F., List, C. (2005) The impossibility of unbiased judgment aggregation. Working paper, London School of Economics

Dietrich, F., List, C. (2006) Judgment aggregation without full rationality. Working paper, London School of Economics

Dietrich, F., List, C. (2007a) Arrow's theorem in judgment aggregation. Social Choice and Welfare 29(1): 19-33

Dietrich, F., List, C. (2007b) Judgment aggregation by quota rules: majority voting generalized. Journal of Theoretical Politics 19(4) (in press)

Dokow, E., Holzman, R. (2005) Aggregation of binary evaluations. Working paper, Technion Israel Institute of Technology

Dokow, E., Holzman, R. (2006) Aggregation of binary evaluations with abstentions. Working paper, Technion Israel Institute of Technology

Gärdenfors, P. (2006) An Arrow-like theorem for voting with logical consequences. Economics and Philosophy 22(2): 181-190

Goodin, R. E., List, C. (2006) Special Majorities Rationalized. British Journal of Political Science 36(2): 213-241

Konieczny, S, Pino-Perez, R. (2002) Merging information under constraints: a logical framework. Journal of Logic and Computation 12: 773-808 
Kornhauser, L. A., Sager, L. G. (1986) Unpacking the Court. Yale Law Journal 96(1): 82-117

List, C., Pettit, P. (2002) Aggregating Sets of Judgments: An Impossibility Result. Economics and Philosophy 18(1): 89-110

List, C., Pettit, P. (2004) Aggregating Sets of Judgments: Two Impossibility Results Compared. Synthese 140(1-2): 207-235

May, K. O. (1952) A set of independent, necessary and sufficient conditions for simple majority decision. Econometrica 20: 680-684

Mongin, P. (2005) Factoring out the impossibility of logical aggregation. Working paper, CNRS, Paris

Nehring, K., Puppe, C. (2002) Strategy-Proof Social Choice on SinglePeaked Domains: Possibility, Impossibility and the Space Between. Working paper, University of California at Davies

Nehring, K., Puppe, C. (2005) The Structure of Strategy-Proof Social Choice: Non-Dictatorship, Anonymity and Neutrality. Working paper, University of Karlsruhe

Nehring, K. Puppe, C. (2006) Conistent Judgement Aggregation: The TruthFunctional Case. Working paper, University of Karlsruhe

Pauly, M., van Hees, M. (2006) Logical Constraints on Judgment Aggregation. Journal of Philosophical Logic 35(6): 569-585

Pettit, P. (2001) Deliberative Democracy and the Discursive Dilemma. Philosophical Issues 11: 268-299

Pigozzi, G. (2006) Belief merging and the discursive dilemma: an argumentbased account to paradoxes of judgment aggregation. Synthese 152(2): 285-298

van Hees, M. (2007) The limits of epistemic democracy. Social Choice and Welfare 28(4): 649-666

\section{A Appendix: proofs}

To prove theorem 1 and corollary 1 , we define a new logic $\mathbf{L}^{*}$ and agenda $X^{*}$ induced by the original logic and agenda as follows.

Informally, each old proposition $p$ in $X$ gives rise to two new propositions $a_{p}, \neg a_{p}$ in $X^{*}$, where $a_{p}$ means " $p$ is accepted", and $\neg a_{p}$ means " $p$ is not accepted" as distinct from " $\neg p$ is accepted" $\left(a_{\neg p}\right)$. If $Y \subseteq \mathbf{L}$ is (in)consistent, so is $\left\{a_{p}: p \in Y\right\}$ in $\mathbf{L}^{*}$. Any old judgment set $A \subseteq X$ induces a new one $A^{*} \subseteq X^{*}$, namely $A^{*}=\left\{a_{p}: p \in A\right\} \cup\left\{\neg a_{p}: p \notin A\right\}$; e.g., the empty judgment set $A=\emptyset$ induces $A^{*}=\left\{\neg a_{p}: p \in X\right\}$. Crucially, $A^{*}$ is always complete, even if $A$ was incomplete; this will allow us to indirectly obtain our results from Dietrich and List's (2006) results on the complete case.

Formally, let $\mathbf{L}^{*}=\left\{a_{p}, \neg a_{p}, \neg \neg a_{p}, \ldots: p \in X\right\}$, a thin propositional language with a single connective $\neg$ ("not") and atomic propositions $a_{p}, p \in X$. A set 
$S^{*} \subseteq \mathbf{L}^{*}$ is consistent ( in $\mathbf{L}^{*}$ ) if accepting all propositions $p \in X$ that $S^{*}$ "says" are accepted is consistent in $\mathbf{L}$ : that is, if the set

$$
\begin{gathered}
S=\left\{p \in X: S^{*} \text { entails } a_{p} \text { in the sense of standard propositional logic }\right\} \\
\left(=\left\{p \in X: S^{*} \text { contains, after cancelling double negations " } \neg \neg ",\right.\right. \\
\left.\left.a_{p} \text { or a pair } a_{q}, \neg a_{q}\right\}\right)
\end{gathered}
$$

is consistent in $\mathbf{L}$. For instance, if $X=\{a, \neg a, a \rightarrow b, \neg(a \rightarrow b), b, \neg b\}$ as in our example, $S^{*}=\left\{a_{a}, a_{a \rightarrow b}, a_{\neg b}\right\}$ is inconsistent (since $S=\{a, a \rightarrow b, \neg b\}$ is inconsistent in $\mathbf{L}$ ), $S^{*}=\left\{a_{a}, a_{a \rightarrow b}, \neg a_{b}\right\}$ is consistent (since $S=\{a, a \rightarrow b\}$ is consistent in $\mathbf{L}$ ), $S^{*}=\left\{a_{b}, \neg a_{\neg b}\right\}$ is consistent (since $S=\{b\}$ is consistent in $\mathbf{L}$ ), and $S^{*}=\left\{a_{b}, \neg a_{b}\right\}$ is inconsistent (since $S=X$ is inconsistent). As is easily verified, the new logic satisfies axioms $\mathrm{C} 1$ to $\mathrm{C} 4$.

Define the new agenda as $X^{*}=\left\{a_{p}, \neg a_{p}: p \in X\right\}$. As $X^{*}$ contains no double negated propositions, the criterion for consistency of a judgment set $S^{*} \subseteq X^{*}$ is simple:

$$
S^{*} \text { is consistent } \Leftrightarrow\left\{\begin{array}{l}
\left\{p \in X: a_{p} \in S^{*}\right\} \text { is consistent in } \mathbf{L} \\
\text { and } S^{*} \text { contains no pair } a_{p}, \neg a_{p} .
\end{array}\right.
$$

We have already defined the notions of non-simplicity and non-separability. For the following lemma, two further agenda conditions are needed. An agenda $X$ is even-number negatable if it has a minimal inconsistent subset $Y$ such that $(Y \backslash Z) \cup\{\neg z: z \in Z\}$ is consistent for some subset $Z \subseteq Y$ of even size; $X$ is locally asymmetric if, for every sub-agenda $Z \subseteq X$, there exists a consistent set $S \subseteq X$ such that $S$, with all the propositions it shares with $Z$ negated (i.e., $(S \backslash Z) \cup\{\neg z: z \in S \cap Z\})$, is inconsistent.

Lemma 1 If the original agenda $X \subseteq \mathbf{L}$ is non-simple and non-separable, then the induced agenda $X^{*} \subseteq \mathbf{L}^{*}$ is non-simple, non-separable, even-number negatable and locally asymmetric.

Proof. Suppose $X$ is non-simple and non-separable. By non-simplicity, there exists a minimal inconsistent subset $Y \subseteq X$ with $|Y| \geq 3$. Let $Y^{*}=\left\{a_{p}: p \in\right.$ $Y\}$. Then $Y^{*} \subseteq X^{*}$ and $\left|Y^{*}\right|=|Y| \geq 3$. By (1) and $Y^{\prime}$ 's minimal inconsistency (in $\mathbf{L}$ ), $Y^{*}$ is minimal inconsistent (in $\mathbf{L}^{*}$ ). So $X^{*}$ is non-simple. By (1) and $Y^{\prime}$ 's minimal inconsistency, $Y^{*}$ becomes consistent by negating one or more of its members $a_{p}$. So $X^{*}$ satisfies the even number negation condition.

To prove local asymmetry, let $Z^{*} \subseteq X^{*}$ be any sub-agenda. We must specify a consistent set $S^{*} \subseteq X^{*}$ that is rendered inconsistent by negating the members shared with $Z^{*}$. As $Z^{*}$ is a non-empty union of pairs $\left\{a_{p}, \neg a_{p}\right\}$, there is at least one pair $\left\{a_{p}, \neg a_{p}\right\} \subseteq Z^{*}$. If also $\left\{a_{\neg p}, \neg a_{\neg p}\right\} \subseteq Z^{*}$, let $S^{*}=\left\{\neg a_{p}, \neg a_{\neg p}\right\}$. By (1), $S^{*}$ is consistent. But after negating the members shared with $Z^{*}$ (i.e., all members) the new set $\left\{a_{p}, a_{\neg p}\right\}$ is inconsistent, also by (1). If $\left\{a_{\neg p}, \neg a_{\neg p}\right\} \nsubseteq Z^{*}$, let $S^{*}=\left\{\neg a_{p}, a_{\neg p}\right\}$. Again, $S^{*}$ is consistent: if it were not, then by (1) $\{\neg p\}$ 
would be inconsistent in $\mathbf{L}$ (i.e., $\neg p$ would be a contradiction in $\mathbf{L}$ ), and so $X$ would be separable (into the sub-agendas $X \backslash\{p, \neg p\}$ and $\{p, \neg p\}$ ), a contradiction. After negating the members $S^{*}$ shares with $Z^{*}$ (i.e., just $\neg a_{p}$ ) the new set $\left\{a_{p}, a_{\neg p}\right\}$ is inconsistent, again by (1). This proves local asymmetry.

To show that $X^{*}$ is non-separable, consider a partition of $X^{*}$ into subagendas $X_{1}^{*}$ and $X_{2}^{*}$. We construct consistent sets $S_{1}^{*} \subseteq X_{1}^{*}$ and $S_{2}^{*} \subseteq X_{2}^{*}$ such that $S_{1}^{*} \cup S_{2}^{*}$ is inconsistent. First suppose there is a $p \in X$ such that $a_{p} \in X_{1}^{*}$ and $a_{\neg p} \in X_{2}^{*}$. Then the sets $S_{1}^{*}:=\left\{a_{p}\right\}$ and $S_{2}^{*}:=\left\{a_{\neg p}\right\}$ are each consistent, by (1) and since no proposition in $X$ is a contradiction (see the argument just made); and the union $S_{1}^{*} \cup S_{2}^{*}=\left\{a_{p}, a_{\neg p}\right\}$ is inconsistent, again by (1). Now suppose there is no $p \in X$ such that $a_{p} \in X_{1}^{*}$ and $a_{\neg p} \in X_{2}^{*}$. Then each of $X_{1}^{*}$ and $X_{2}^{*}$ is a non-empty union of quadruples $\left\{a_{p}, \neg a_{p}, a_{\neg p}, \neg a_{\neg p}\right\}$. So each of $X_{1}:=\left\{p \in X: a_{p} \in X_{1}^{*}\right\}$ and $X_{2}:=\left\{p \in X: a_{p} \in X_{2}^{*}\right\}$ is a non-empty union of pairs $\{p, \neg p\} \subseteq X$, hence a sub-agenda of $X$. Moreover, $X_{1}, X_{2}$ form a partition of $X: X_{1} \cap X_{2}=\emptyset$ by $X_{1}^{*} \cap X_{2}^{*}=\emptyset$, and $X_{1} \cup X_{2}=X$ by $X_{1}^{*} \cup X_{2}^{*}=X^{*}$. So, as $X$ is non-separable, there are sets $S_{1} \subseteq X_{1}$ and $S_{2} \subseteq X_{2}$ that are (in L) consistent with inconsistent union $S_{1} \cup S_{2}$. It follows, by (1), that the sets $S_{1}^{*}:=\left\{a_{p}: p \in S_{1}\right\}\left(\subseteq X_{1}^{*}\right)$ and $S_{2}^{*}:=\left\{a_{p}: p \in S_{2}\right\}\left(\subseteq X_{2}^{*}\right)$ are each consistent and have inconsistent union $S_{1}^{*} \cup S_{2}^{*}\left(=\left\{a_{p}: p \in S_{1} \cup S_{2}\right\}\right)$, as required.

For the next lemma, a judgment set is fully rational if it is consistent and complete. An aggregation function is fully rational if it produces a fully rational judgment set as output; it has universal domain if it accepts the profiles of fully rational individual judgment sets as input.

Lemma 2 The assignment $A \longmapsto \bar{A}:=\left\{a_{p}: p \in A\right\} \cup\left\{\neg a_{p}: p \notin A\right\}$ defines a bijection between the consistent judgment sets $A \subseteq X$ and the fully rational judgment sets $A^{*} \subseteq X^{*}$, with inverse given by $A^{*} \longmapsto \underline{A^{*}}:=\left\{p: a_{p} \in A^{*}\right\}$. In particular, any consistent full-domain aggregation function $F$ for $X$ induces a fully rational universal-domain aggregation function $F^{*}$ for $X^{*}$, given by

$$
F^{*}\left(A_{1}^{*}, \ldots, A_{n}^{*}\right):=\overline{F\left(\underline{A_{1}^{*}}, \ldots, \underline{A_{n}^{*}}\right)} .
$$

Proof. For all consistent $A \subseteq X$, the set $\bar{A} \subseteq X^{*}$ is by definition complete and by (1) consistent, hence fully rational. The assignment is injective: if consistent sets $A, B \subseteq X$ are distinct, so are $\bar{A}$ and $\bar{B}$. It is also surjective: every fully rational set $A^{*} \subseteq X^{*}$ is the image of some consistent set $A \subseteq X$, namely of the set $\underline{A^{*}}$, since

$$
\begin{aligned}
\overline{\left(A^{*}\right)} & =\left\{a_{p}: p \in\left\{q: a_{q} \in A^{*}\right\}\right\} \cup\left\{\neg a_{p}: p \notin\left\{q: a_{q} \in A^{*}\right\}\right\} \\
& =\left\{a_{p}: a_{p} \in A^{*}\right\} \cup\left\{\neg a_{p}: a_{p} \notin A^{*}\right\} \\
& =\left\{a_{p}: a_{p} \in A^{*}\right\} \cup\left\{\neg a_{p}: \neg a_{p} \in A^{*}\right\} \text { (as } A^{*} \text { is fully rational) } \\
& =A^{*} .
\end{aligned}
$$

The latter also shows that $A^{*} \longmapsto \underline{A^{*}}$ is the inverse assignment. 
The next lemma's unbiasedness condition is defined in section 4 (by taking $p=\neg q$ in the definition of systematicity).

Lemma 3 In Lemma $2, F^{*}$ is unbiased if and only if $F$ is acceptance/rejection neutral.

Proof. First, let $F$ be acceptance/rejection neutral. To show that $F^{*}$ is unbiased, take any pair $a_{p}, \neg a_{p} \in X^{*}$ and any profiles $\left(A_{1}^{*}, \ldots, A_{n}^{*}\right),\left(B_{1}^{*}, \ldots, B_{n}^{*}\right)$ admissible for $F^{*}$ such that, for all $i, a_{p} \in A_{i}^{*} \Leftrightarrow \neg a_{p} \in B_{i}^{*}$, hence (by $B_{i}^{*}$ 's full rationality) $a_{p} \in A_{i}^{*} \Leftrightarrow a_{p} \notin B_{i}^{*}$. So, for all $i, p \in A_{i}^{*} \Leftrightarrow p \notin B_{i}^{*}$. So, as $F$ is acceptance/rejection neutral, $p \in F\left(A_{1}^{*}, \ldots, A_{n}^{*}\right) \Leftrightarrow \bar{p} \notin F\left(B_{1}^{*}, \ldots, B_{n}^{*}\right)$. Hence, by definition of $F^{*}, a_{p} \in F^{*}\left(A_{1}^{*}, \ldots, \overline{A_{n}^{*}}\right) \Leftrightarrow \overline{\neg a_{p}} \in F^{*}\left(B_{1}^{*}, \bar{\ldots}, B_{n}^{*}\right)$, as required.

To prove the converse, note first that $F$ is is retrievable from $F^{*}$ via

$$
F\left(A_{1}, \ldots, A_{n}\right)=F^{*}\left(\overline{A_{1}}, \ldots, \overline{A_{n}}\right) .
$$

Now let $F^{*}$ be unbiased. To show that $F$ is acceptance/rejection neutral, consider a $p \in X$ and admissible profiles $\left(A_{1}, \ldots, A_{n}\right),\left(B_{1}, \ldots, B_{n}\right)$ such that, for all $i, p \in A_{i} \Leftrightarrow p \notin B_{i}$. Then, for all $i, a_{p} \in \overline{A_{i}} \Leftrightarrow \neg a_{p} \in \overline{B_{i}}$. So, as $F^{*}$ is unbiased, $a_{p} \in F^{*}\left(\overline{A_{1}}, \ldots, \overline{A_{n}}\right) \Leftrightarrow \neg a_{p} \in F^{*}\left(\overline{B_{1}}, \ldots, \overline{B_{n}}\right)$, hence (by $F^{*}$ 's full rationality) $a_{p} \in F^{*}\left(\overline{A_{1}}, \ldots, \overline{A_{n}}\right) \Leftrightarrow a_{p} \notin F^{*}\left(\overline{B_{1}}, \ldots, \overline{B_{n}}\right)$. So, by (2) applied to the profiles $\left(A_{1}, \ldots, A_{n}\right)$ and $\left(B_{1}, \ldots, B_{n}\right), p \in F\left(A_{1}, \ldots, A_{n}\right) \Leftrightarrow p \notin F\left(B_{1}, \ldots, B_{n}\right)$, as required.

Lemma 4 In Lemma $2, F^{*}$ is a dictatorship if and only if $F$ is.

Proof. If $F$ is a dictatorship, say with dictator $i, F^{*}$ is given by

$$
F^{*}\left(A_{1}^{*}, \ldots, A_{n}^{*}\right)=\overline{\left(A_{i}^{*}\right)}=A_{i}^{*},
$$

hence is a dictatorhip. To prove the converse, recall (from the proof of lemma 3 ) that $F$ is given by (2). So if $F^{*}$ is a dictatorship, say with dictator $i, F$ is given by

$$
F\left(A_{1}, \ldots, A_{n}\right)=\underline{\left(\overline{A_{i}}\right)}=A_{i},
$$

hence is a dictatorship.

Using the above lemmas, we derive Theorem 1 from the following result.

Theorem 2 (Dietrich and List 2005, theorem 2) For a non-simple, non-separable, even-number negatable and locally asymmetric agenda, every fully rational universal-domain aggregation function satisfying unbiasedness is a dictatorship. 
Proof of theorem 1. Take any non-simple and non-separable agenda $X$ and consider any consistent full-domain aggregation function $F$ satisfying acceptance/rejection neutrality. By lemma 1 , the induced agenda $X^{*}$ is non-simple, non-separable, even-number negatable and locally asymmetric. Take the induced aggregation function $F^{*}$ of lemma 2. By lemmas 2 and $3, F^{*}$ is fully rational, universal-domain and unbiased, hence by theorem 2 a dictatorship. By lemma $4, F$ is therefore also a dictatorship, as required. To prove that the agenda condition of non-simplicity and non-separability in theorem 1 is tight (for $n \geq 3$ ), note that if $X$ violates non-simplicity majority voting (with full domain) among a given odd-sized non-singleton subset of $N$ is consistent, as well as acceptance/rejection neutral and non-dictatorial; and if $X$ is separable, say into sub-agendas $X_{1}$ and $X_{2}$, the full-domain aggregation function that makes some individual dictatorial on $X_{1}$ and another one dictatorial on $X_{2}$ is consistent, as well as acceptance/rejection neutral and non-dictatorial.

The next lemma's independence condition is defined in section 4 (by taking $p=q$ in the definition of systematicity)

Lemma 5 Any consistent full-domain aggregation function satisfying acceptance/rejection neutrality also satisfies independence.

Proof. Let $F$ be a consistent full-domain aggregation function satisfying acceptance/rejection neutrality. By lemmas $2-3, F$ induces a fully rational universaldomain unbiased aggregation function $F^{*}$ for $X^{*}$. So $F^{*}$ is independent by lemma 1 in Dietrich and List (2005). To show that $F$ is independent too, take any proposition $p \in X$ and profiles $\left(A_{1}, \ldots, A_{n}\right),\left(B_{1}, \ldots, B_{n}\right)$ admissible for $F$, such that, for all $i, p \in A_{i} \Leftrightarrow p \in B_{i}$. Then, for all $i, a_{p} \in \overline{A_{i}} \Leftrightarrow a_{p} \in \overline{B_{i}}$. Thus, as $F^{*}$ is independent, $a_{p} \in F^{*}\left(\overline{A_{1}}, \ldots, \overline{A_{n}}\right) \Leftrightarrow a_{p} \in F^{*}\left(\overline{B_{1}}, \ldots, \overline{B_{n}}\right)$. So, using (2) as in earlier proofs, $p \in F\left(A_{1}, \ldots, A_{n}\right) \Leftrightarrow p \in F\left(B_{1}, \ldots, B_{n}\right)$, as required.

Proof of corollary 1. Consider any consistent full-domain aggregation function $F$ satisfying acceptance/rejection neutrality. Take any non-simple and non-separable sub-agenda $Y \subseteq X$ (if there exists none, the result vacuously holds). By lemma 5, $F$ is independent, hence induces a well-defined full-domain aggregation function $F^{Y}$ for the sub-agenda $Y . F^{Y}$ inherits from $F$ its consistency and acceptance/rejection neutrality. Thus theorem 1 applies to $F^{Y}$, and corollary 1 follows immediately. 